\title{
latrogenic aortopulmonary fistula after balloon dilation of right pulmonary artery stent following truncus arteriousus type 1 repair
}

\begin{abstract}
latrogenic aortopulmonary (AP) fistula is an unusual complication after balloon pulmonary artery (PA) angioplasty or branch pulmonary arteries (PAs) stent dilation. The majority of the cases were reported after transcatheter interventions on the PAs or right ventricular outflow tract (RVOT), in patients with transposition of great arteries (TGA) who underwent PA angioplasty following arterial switch operation (ASO) or after transcutaneous pulmonary valve replacement in patients who had undergone a Ross procedure [1].We present a case of iatrogenic AP fistula in a 4-year-old patient with a history of repaired truncus arteriosus type 1 followed by bilateral PAs stent placement and balloon dilation,17 months later redilation of the right pulmonary artery (RPA) stent resulted in a AP fistula with significant left to right shunt which was successfully closed with a covered stent. This case demonstrates a previously unreported complication of transcatheter RPA stent reintervention after truncus arteriosus repair.
\end{abstract}

Keywords:

Aortopulmonary fistula; Pulmonary artery stent; Catheter intervention

\section{Case Report}

A 4 years old male patient diagnosed with having truncus arteriosus type 1 in neonatal period underwent truncus arteriosus repair with placement of right ventricular to pulmonary artery (RV-PA) conduit contegra size $12 \mathrm{~mm}$ at the age of 2 months. Thereafter at the age of 11 months he developed severe RV-PA conduit and bilateral branch PAs stenosis and underwent conduit change with pulmonary homograft size $19 \mathrm{~mm}$ and reconstruction of PAs. Over a period of 17 months a serial transthoracic echo showed progressive severe branch PAs stenosis. Cardiac catheterization hemodynamics showed RVSP of $63 \mathrm{mmHg}$, systemic pressure 101/63 and PAs angiographic study showed severe RPA and LPA stenosis. Implantation of RPA and LPA stents (Cordis size $10 \mathrm{~mm}$ X $19 \mathrm{~mm}$ ) were successfully performed, the RVSP dropped to $34 \mathrm{mmHg}$ and PAs angiography showed both stents in good position with no stenosis. After 1 year echo showed branch PAs stenosis with calculated RPA and LPA maximum pressure gradient of $50 \mathrm{mmHg}$ so underwent diagnostic catheterization. The hemodynamics showed RVSP: $55 \mathrm{mmHg}$ and systemic blood pressure: $110 \mathrm{mmHg}$ ), the pressure gradient across RV-PA conduit was $10 \mathrm{mmHg}$, RPA: $22 \mathrm{mmHg}$ and LPA: $13 \mathrm{mmHg}$. Branch PAs angiography showed significant endothelial growth with severe narrowing both stents in RPA (the narrowest area measured to be $4.7 \mathrm{~mm}$ ) and LPA (the narrowest area measured to be $6.3 \mathrm{~mm}$ ), so it was decided to balloon dilate both stents. Six (6) french sheath was exchanged to seven (7) french sheath. Atlas balloon size $12 \mathrm{~mm}$ X $20 \mathrm{~mm}$ was used and inflated twice in the LPA then same balloon inflated twice for the RPA. Thereafter, sudden hemodynamic changes had been noticed with increase RPA pressure from $25 \mathrm{mmHg}$ pulse pressure 100/31 mmHg, Aortogram showed a communication between RPA and aorta with significant leak with left to right shunt (Figure 1). A CP covered stent $22 \mathrm{~mm}$ length was successfully deployed over balloon in balloon size $12 \mathrm{~mm}$ X $30 \mathrm{~mm}$ in the RPA. Subsequent aortogram showed no leak and the stent was in good position (Figure 2). to $49 \mathrm{mmHg}$ and there was wide systemic
Sultan A. Alharthi*, Atif Alsahari, Falih A. AlQahtani, Jassim M. Abdulhamed

Department of Pediatric Cardiology, Prince Sultan Cardiac Center, Riyadh, Kingdom of Saudi Arabia

*Author for Corresponding: Tel: 00966556225788

Email:salharthi3@gmail.com

Received date: Nov 13, 2018

Accepted date: Jan 02, 2019

Publication date: Jan 12, 2019 


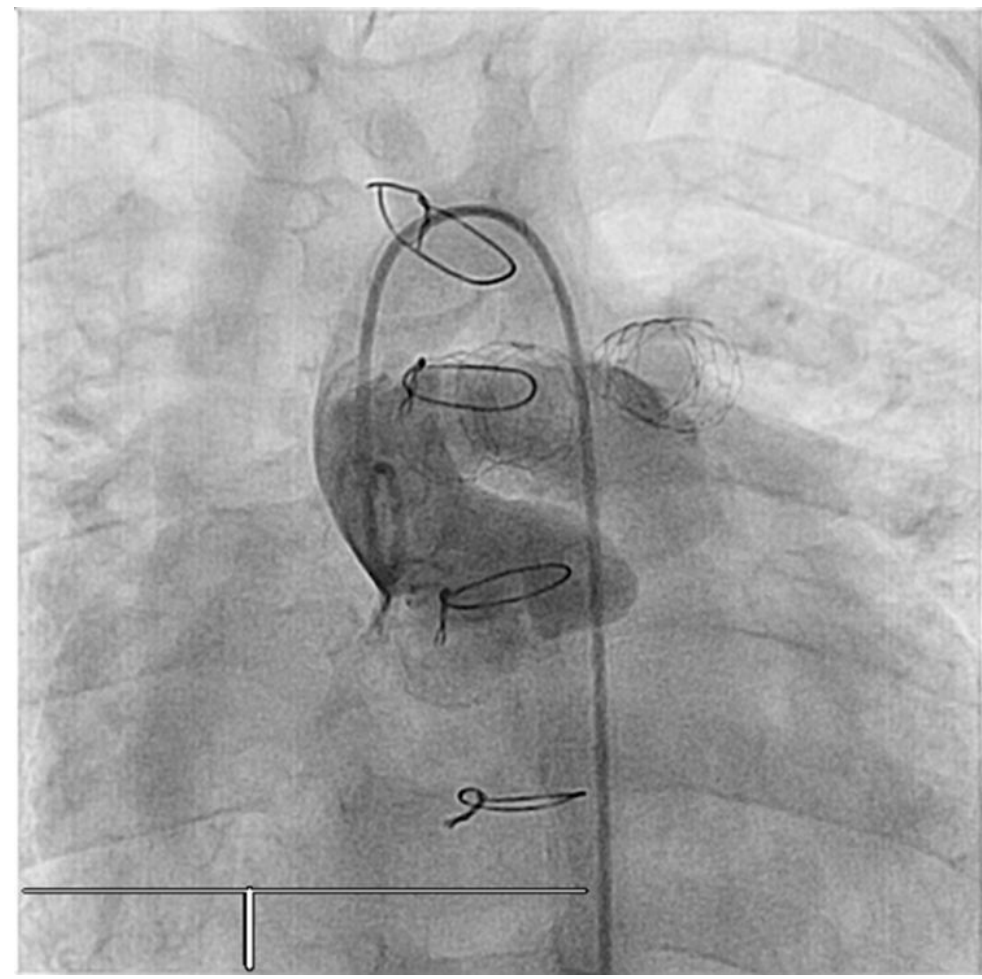

Figure 1: straight posterior-anterior view of the aortogram with pigtail catheter following the RPA stent dilation, demonstrating dye in the PAs indicating, AP communication.

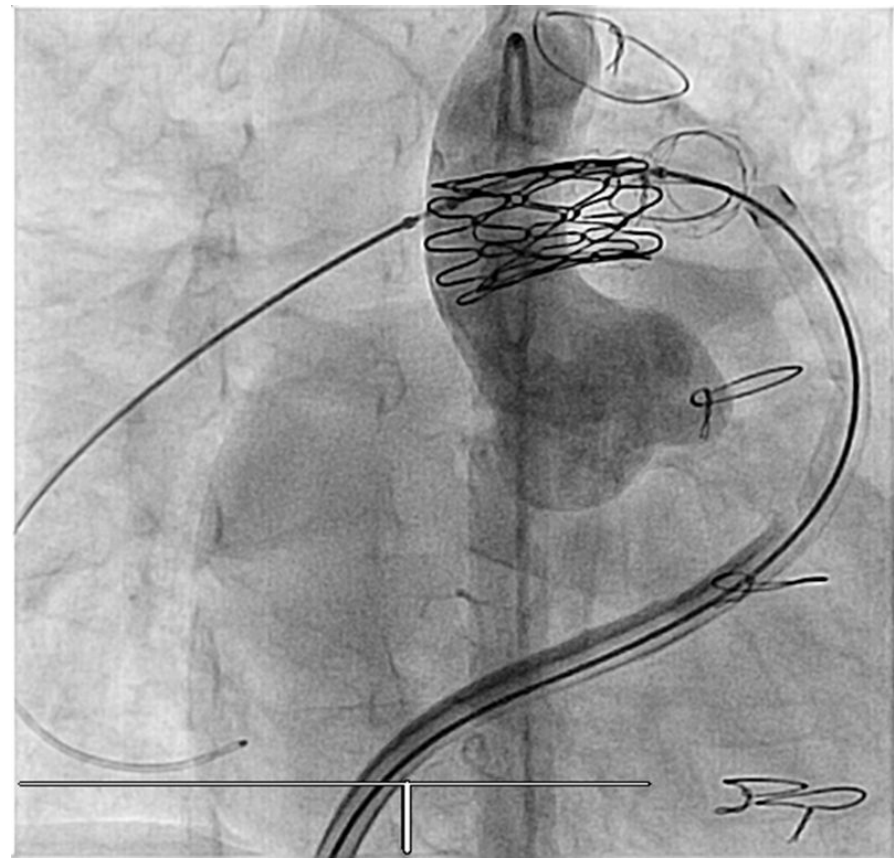

Figure 2: Aortogram, RAO 27 degree view following CP covered stent implantation in the RPA showing complete closure of the AP communication.

\section{Discussion}

Patients with truncus arteriosus often require PAs and/or RVOT reintervention within the first year of repair [1]. While in TGA, PAs stenosis may occur in 4-28\% of patients after an ASO. Balloon dilation can be attempted with variable results, while stenting is a more definitive option when balloon dilation fails [2].
The majority of the iatrogenic AP fistulas cases were reported after transcatheter interventions on the PAs or RVOT in patients with TGA who underwent PA angioplasty after an ASO and most of these cases were managed by transcatheter intervention with use of covered stents or endovascular stent graft [3]. One case was reported in a patient with truncus arteriosus repaired without the use of RV-PA conduit; later on 
RVOT stent was used. Redilation of the stent resulted in AP communication and then managed surgically [4]. In our case the RPA stent was over dilated until it was extended to the wall of the aorta and ruptured and subsequently it created a communication between both arteries with significant left to right shunt. We observed from the previous case reports and our case that iatrogenic AP fistula had occurred more frequently after an intervention when the aorta and PA or branch PAs became anatomically closer to each other, which can explain why the majority of reported cases happened post TGA repair where the lecompte procedure brings the aorta and PA anatomically closer to each other. We are proposing that when a patient at a higher risk of iatrogenic AP fistula due to the anatomically close relationship between the aorta and PA or its branches, careful balloon angioplasty and stents dilation should be considered A suggested approach is to do simultaneous angiogram in the aorta and PA to measure the distance between both arteries to see the extent of pulmonary balloon dilation.

\section{Conclusion}

Iatrogenic AP fistula is a rare complication after balloon pulmonary artery angioplasty or branch PAs stent dilation. When the patient is at higher risk for an iatrogenic AP fistula due to anatomically close relationship between the aorta and $\mathrm{PA}$ and or its branches like post TGA arterial switch and truncus arteriosus repair a simultaneous angiogram in the aorta and PA to measure the distance between both arteries and then careful PA balloon angioplasty and stent dilation should be considered.

\section{References}

1. Lund AM, Vogel M, Marshall AC, et al. Early reintervention on the pulmonary arteries and right ventricular outflow tract after neonatal or early infant repair of truncus arteriosus using homograft conduits. Am J Cardiol. 108: 106-13 (2011)

2. Tzifa A, Papagiannis J, Qureshi S. Iatrogenic aortopulmonary window after balloon dilation of left pulmonary artery stenosis following arterial switch operation. J Invasive Cardiol. 25: E188-90 (2013).

3. Torres A, Sanders SP, Vincent JA, et al. Iatrogenic aortopulmonary communications after transcatheter interventions on the right ventricular outflow tract or pulmonary artery: Pathophysiologic, diagnostic, and management considerations. Catheter Cardiovasc Interv. 86: 438-52 (2015).

4. Bernier PL, Hallbergson A, Schachtner SK, et al. Aortopulmonary fistula after outflow tract stent in repaired truncus. Ann Thorac Surg. 98: e55-7 (2014). 